\title{
SIGNATURES OF COOLING FLOWS
}

\author{
A.C. Fabian \\ Institute of Astronomy \\ Madingley Road \\ Cambridge CB3 0HA \\ U.K.
}

\begin{abstract}
The evidence for cooling flows in clusters of galaxies is discussed. Peaked Xray surface brightness profiles and the presence of soft X-ray spectral components are characteristic signatures of cooling flows. The best available data are consistent with gas cooling at a rate of $\sim 100 \mathrm{M}_{\odot} \mathrm{yr}^{-1}$ and depositing cooled matter over a radius of $100-200 \mathrm{kpc}$. The same rate is obtained from observations of gas cooling over a range of temperatures and thus a range of cooling times covering less than $3 \times 10^{7} \mathrm{yr}$ to more than $3 \times 10^{9} \mathrm{yr}$. It appears that cooling flows are both steady and long-lived. Some indirect optical evidence for distant cooling flows $(0.5<z<1)$ is presented.
\end{abstract}

\section{INTRODUCTION}

Cooling flows appear to be relatively common in the centres of the hot gaseous atmospheres of clusters and groups of galaxies. They also occur in many early-type galaxies. The gas is densest in the core of a cluster and its cooling time, $t_{c o o l}$, due to the emission of X-rays such as those observed, is shortest there. A cooling flow is formed when $t_{\text {cool }}$ is less than the age of the system, $t_{a}\left(\sim H^{-1}\right)$.

In the cases considered here, $t_{\text {cool }}$ exceeds the gravitational free-fall time, $t_{\text {grav }}$, within the cluster (except perhaps in some very small region at the centre), so, for a cooling flow,

$$
t_{a}>t_{\text {cool }}>t_{\text {grav }}
$$

The flow takes place because the gas density has to rise to support the weight of the overlying gas.

If that is not immediately clear, consider the gaseous atmosphere trapped in the gravitational potential well of the cluster or galaxy to be divided into two parts at the radius, $r_{\text {cool }}$, where $t_{\text {cool }}=t_{a}$. The gas pressure at $r_{\text {cool }}$ is determined by the weight of the overlying gas, in which cooling is not important. Within $r_{\text {cool }}$, cooling is tending to reduce the gas temperature and so the gas density must rise in order to maintain the pressure at $r_{\text {cool }}$. The only way for the density to rise (ignoring matter sources within $r_{\text {cool }}$, which is a safe assumption in a cluster of galaxies) is for the gas to flow inward. This is the cooling flow.

If the initial gas temperature exceeds the virial temperature of the central galaxy (this is generally the case for rich clusters but not for poor ones or individual galaxies) then the gas does cool as it flows in. When the temperature has dropped to the virial temperature of the central galaxy, the gas then heats up as it flows further in due to the 
release of gravitational energy. The gas temperature eventually drops catastrophically in the core of the galaxy if its gravitational potential flattens there. All this means that the gas within $r_{\text {cool }}$ radiates its thermal energy, plus $P d V$ work and gravitational energy released in the flow.

This is how an idealized, homogeneous cooling flow, in which the gas has a unique temperature and density at each radius, will behave. Observations of real cooling flows shows that they are inhomogeneous and must consist of a mixture of temperatures and densities at each radius. The homogeneous flow is, however, still a fair approximation of the mean flow.

In this review, I concentrate on the $\mathrm{X}$-ray evidence for cooling flows and their implications for central cluster galaxies. A brief discussion of the optical appearance of cooling flows is provided and used to suggest that they were widespread at redshifts of 0.5 and more. Apart from a short comment, I ignore any continuous heat source capable of disrupting a flow since the $\mathrm{X}$-ray data show that there are no such sources. An alternative view is given in the following paper by $W$. Tucker (this Volume).

\section{X-RAY EVIDENCE FOR COOLING FLOWS}

\section{$2 a X$-ray Images}

A sharply-peaked X-ray surface brightness distribution is often indicative of a cooling flow. It shows that the gas density is rising steeply towards the centre of the cluster or group which means either that the gas pressure is rising steeply or, if the pressure is roughly constant, that the gas temperature is falling. The equation of hydrostatic equilibrium

$$
\frac{d P_{g a s}}{d r}=-\rho_{g a s} g
$$

shows that the first option requires a large gravitational field with a virial temperature for that region which exceeds that of the gas. This is not the case within the core of a cluster of galaxies where the potential is flattening off *. The constant pressure option is the simplest one consistent with the data (Fabian et al. 1981; Stewart et al. 1984b).

Most of the images have been obtained with the Einstein Observatory and with EXOSAT, although the peaks were anticipated with data from the Copernicus satellite (Fabian et al.1974; Mitchell et al. 1975), from rocket-borne telescopes (Gorenstein et al. 1977) and with the modulation collimators on SAS 3 (Helmken et al. 1978).

Deprojection, or modelling, of the X-ray images shows that $t_{\text {cool }}<H_{0}^{-1}$ within the central region of more than 30 to 50 per cent of the clusters well-detected with the Einstein Observatory (Stewart et al. 1984b; Arnaud 1988). Whether $H_{0}^{-1}$ should be used for $t_{a}$ is debatable (but see $\S 2 \mathrm{~b}$ ), and it is not obvious how to extrapolate from 'well-detected' clusters to all clusters. Inspection of the results shows however that reducing $t_{a}$ by 2 , say, does not much change the fraction of clusters which contain cooling flows, and it must be remembered that the spatial resolution of the commonly-used IPC was not sufficient to resolve the central regions of many clusters. In those cases, the measured $t_{\text {cool }}$ is just an upper limit. The overall picture is that the prime criterion for a cooling flow, $t_{\text {cool }}<10^{10} \mathrm{yr}$, is satisfied in a large fraction of clusters. It is also satisfied in a number of poor clusters

* Jones \& Forman (1984) modelled the emission as due to gas at constant temperature in the potential well of the cluster. They found that a 'central excess' of emission is required in the cooling flow cases. 


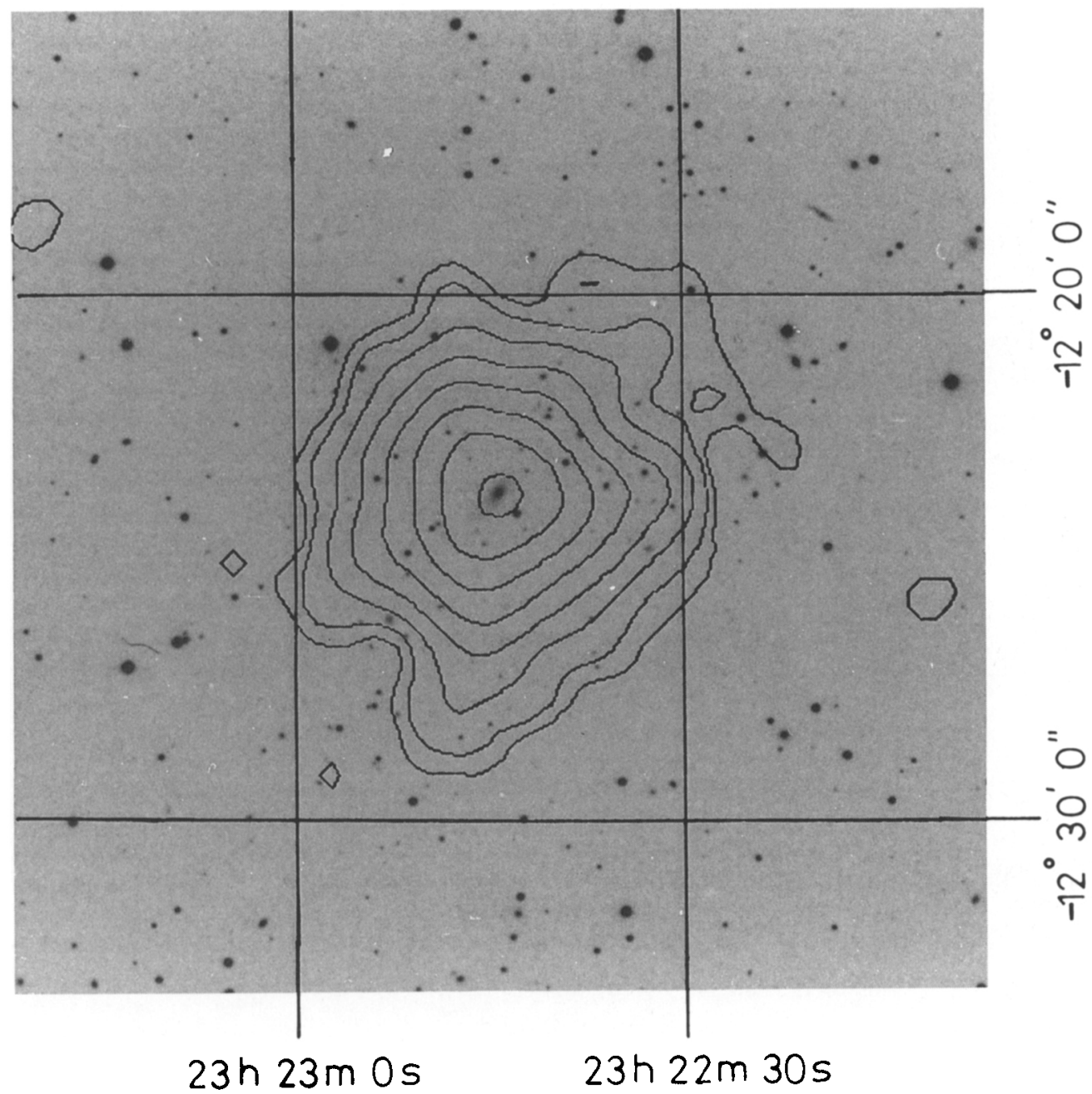

Figure 1. IPC X-ray surface brightness contours of A2597 superimposed on an optical image of the cluster. Note that the contours peak onto the central cluster galaxy. The mass deposition rate in $\mathrm{A} 2597$ is $\sim 370 \mathrm{M}_{\odot} \mathrm{yr}^{-1}$ (Crawford et al. 1989).

and groups (Schwartz, Schwarz \& Tucker 1980; Canizares, Stewart \& Fabian 1983; Singh, Westergaurd \& Schnopper 1986). The implication of these statistics is that cooling flows are both common and long-lived,

The mass deposition rate, $\dot{M}$, due to cooling (i.e. the accretion rate, although I resist using that term since most of the gas does not change its radius very much) can be estimated from the X-ray images by using the luminosity associated with the cooling region (i.e. $L_{\text {cool }}$ within $r_{\text {cool }}$ ) and assuming that it is all due to the radiation of the thermal 
Cooling Spectrum at $10 \mathrm{eV}$ Resolution

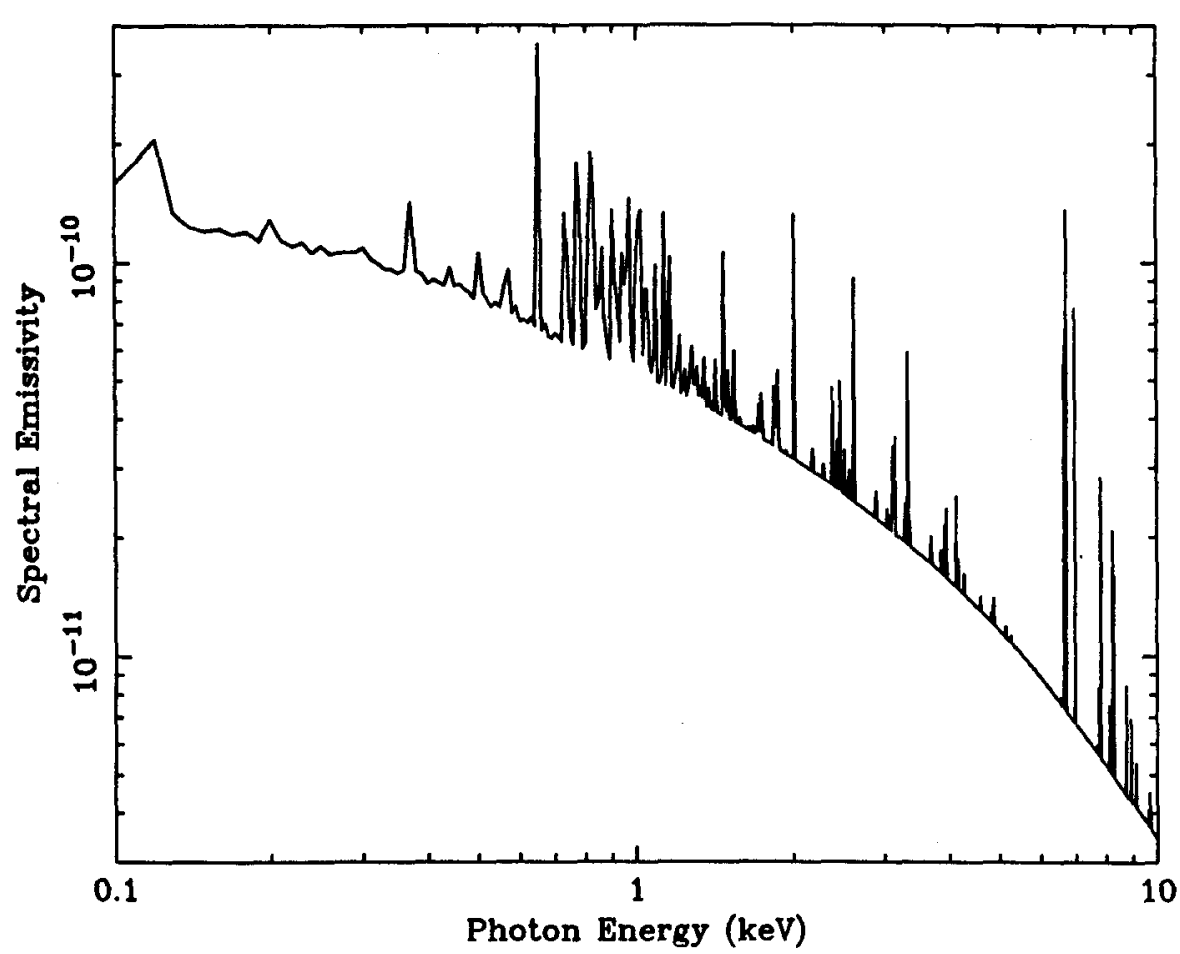

Figure 2. X-ray spectrum at $10 \mathrm{eV}$ resolution of gas cooling from $6 \mathrm{keV}$.

energy of the gas, plus the $P d V$ work done.

$$
L_{\text {cool }}=\frac{5}{2} \frac{\dot{M}}{\mu m} k T,
$$

where $T$ is the temperature of the gas at $r_{\text {cool }}$. Values of $\dot{M}=50-100 \mathrm{M}_{\odot} \mathrm{yr}^{-1}$ are fairly typical for cluster cooling flows. Some clusters show $\dot{M} \sim 500 \mathrm{M}_{\odot} \mathrm{yr}^{-1}$ (e.g. PKS0745, A1795, A2597 and Hydra A).

Since we often measure a surface brightness profile for the cluster core (where it is well-resolved), we have $L_{\text {cool }}(r)$ which can be turned into $\dot{M}(r)$, the mass deposition rate within radius $r$. Generally, we find

$$
\dot{M}(r) \propto r .
$$

The surface brightness profiles are less peaked than they would be if all the gas were to flow to the centre. This means that the gas must be inhomogeneous, so that some of the gas cools out of the flow at large radii and some continues to flow in. The actual computation of $\dot{M}(r)$ is in detail complicated, since we need to take into account how the gas cools and 


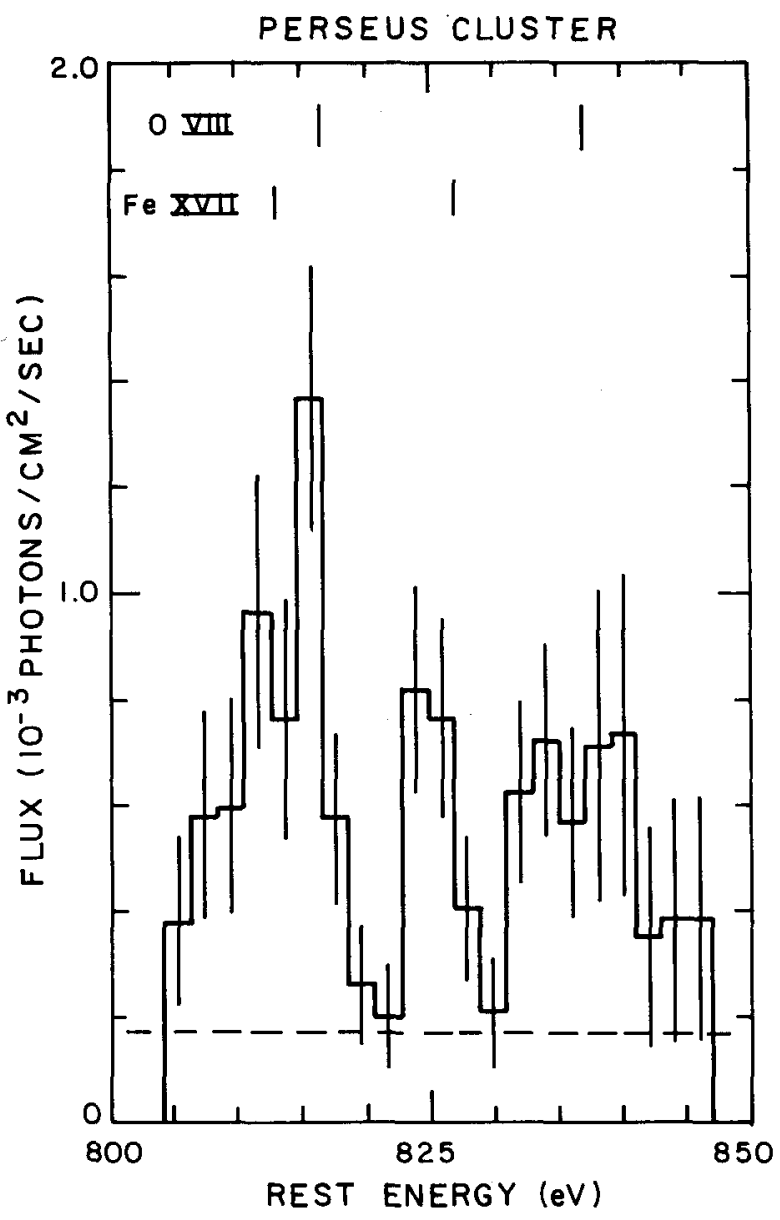

Figure 3. Part of FPCS spectrum of the Perseus cluster. Note the prominent emission lines of OVIII and Fe XVII (from Canizares, Markert \& Donahue 1988). The emission measure of the gas producing the Fe XVII lines is consistent with about $200 \mathrm{M}_{\odot} \mathrm{yr}^{-1}$ of gas cooling through $\sim 5.10^{6}-10^{6} \mathrm{~K}$. This is the $\dot{M}$ found from imaging and SSS studies, which are most sensitive to higher temperature gas (typically $5.10^{6}-3.10^{7} \mathrm{~K}$ ).

any gravitational work done, but since plain cooling dominates, a simple analysis gives a fair approximation to the profile (see Fabian, Arnaud \& Thomas 1986; Thomas, Fabian \& Nulsen 1987; White \& Sarazin 1987).

\section{2b X-ray Spectra}

Key evidence that the gas does actually cool is given by moderate to high resolution spectra of the cluster cores. Canizares et al. (1979, 1982), Canizares (1981); Mushotzky et al. (1981) and Lea et al. (1983) used the Focal Plane Crystal Spectrometer (FPCS) and the Solid State Spectrometer (SSS) on the Einstein Observatory to show that there are low temperature 
components in the Perseus and Virgo clusters, consistent with the existence of cooling flows. Detailed examination of the line fluxes and of the emission measures of the cooler gas by Canizares, Markert \& Donahue (1988) and Mushotzky \& Szymkowiak (1988) shows that, in the case of the Perseus cluster, the gas loses at least 90 per cent of its thermal energy and that the mass deposition rates are in agreement with those obtained from the images. Good agreement is obtained also in several other clusters. The SSS results show that the emission measures vary with temperature in the manner expected from a cooling gas. The importance of these data cannot be overemphasized since they show that the gas does cool. Any 'alternative interpretation' of the images must confront this spectroscopic evidence successfully.

The cooling time of the gas in the Perseus cluster which emits the FeXVII line $\left(T<5 \times 10^{6} \mathrm{~K}\right)$ is less than $3 \times 10^{7} \mathrm{yr}$. Since the emission measure of this gas agrees with that inferred from the gas cooling at the higher temperatures which dominate the images and the SSS result, we must conclude that the flow is steady (Nulsen 1988). The shape of the continuum and line spectrum observed with the SSS is consistent with the same mass deposition rate at all X-ray temperatures (as expected) so we must again conclude that the flow is long-lived. It cannot be some intermittent or transient phenomenon only a billion years old.

\section{2c Summary}

The overwhelming evidence of the images and spectra shows that cooling does occur at a steady rate over long times (at least several billion years). Since mass is then cooling out of the hot phase at rates of hundreds of solar masses per year an inflow must occur. We do not expect yet to have direct evidence of any inward flow since the velocity is highly subsonic at $\sim 10 \mathrm{~km} \mathrm{~s}^{-1}$.

Cooling flows are common and all of the nearest clusters (Virgo, Centaurus, Fornax, Perseus, Ophiuchus...) contain one apart from the Coma cluster. Even that could contain a disrupted flow (Fabian, Nulsen \& Canizares 1984). Many flows are observed out to a redshift of 0.1, with more distant ones being in 3C295 (Henry \& Henriksen 1987) and 1 E0839 (Wolter et al., this Volume).

The values of $\dot{M}$ are probably good to a factor of 2 (Arnaud 1988) and could be higher if there are denser blobs beyond $r_{\text {cool }}$ (Thomas et al. 1987).

\section{The Fate of the Cooled Gas}

The accumulated mass of cooled gas can be considerable;

$$
\dot{M} H_{0}^{-1}=2 \times 10^{12}\left(\frac{\dot{M}}{100 \mathrm{M}_{\odot} \mathrm{yr}^{-1}}\right) \dot{\mathrm{M}}_{\odot}
$$

This is a significant fraction of the mass of the central galaxy. It suggests that we are witnessing the continued formation of that galaxy, which is typically one of the largest galaxies known.

If the gas forms stars, then cooling flows are the largest and strongest regions of star formation in our part of the Universe. Even a casual comparison of a central cluster galaxy and a spiral galaxy such as our own, which is thought to be forming stars at a rate of $3-10 \mathrm{M}_{\odot} \mathrm{yr}^{-1}$, shows that cooling flows must form low-mass stars (Fabian, Nulsen 


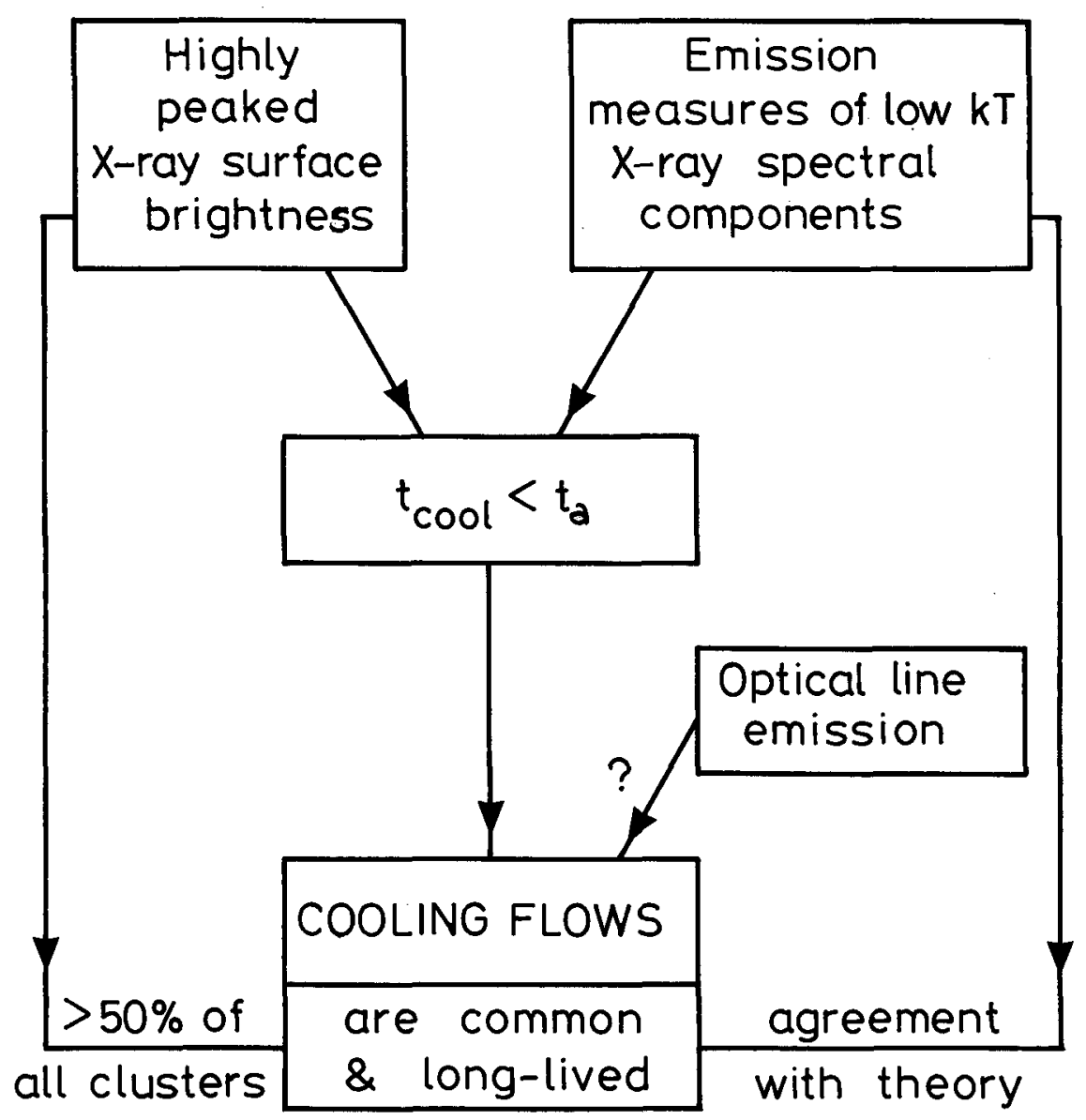

Figure 4. The evidence for Cooling Flows.

\& Canizares 1982; Sarazin \& O'Connell 1983). Massive stars would make central cluster galaxies much bluer than they are.

It should be stressed that the cooling gas is not directly detected once it has cooled below about $3 \times 10^{6} \mathrm{~K}$. If it recombines and forms low-mass stars $\left(\left\langle M_{*}\right\rangle<0.5 \mathrm{M}_{\odot}\right)$ in a distributed manner $(M(r) \propto r)$ then there is no reason for it to have been seen. The optical line luminosity from the gas and the continuum from the stars are undetectable with current means.

There is, however, plenty of evidence for dark matter in clusters and low-mass stars are one plausible form of dark matter. The manner of the mass deposition with radius, $\dot{M}(r) \propto r$, leads to an isothermal halo which is consistent with the dark matter distribution around large galaxies (e.g. M87, Stewart et al. 1984a; Mould et al. 1987). Cooling flows are a source of baryonic dark matter. 


\section{3b Heating}

Since the implied star formation rates are so large and there is little sign of it optically, there have been a number of studies suggesting that the rates have been grossly over-estimated. Some heat source that balances the cooling is the obvious solution. Cosmic rays Tucker \& Rosner 1982), conduction (Bertschinger \& Meiksin 1986), supernovae (Silk et al. 1986) and galaxy motions (Miller 1986) have all been invoked as heat sources. Unfortunately for these models, the X-ray spectra indicate cooling without heating. None of the models proposed so far is able (or even attempts!) to account for the X-ray line emission. There are other problems with these heat sources as well (see Fabian 1988; Bregman \& David 1988).

The total level of heating necessary is very large, $\sim 10^{62} \mathrm{erg}$ for a large flow over $t_{a}$ and so if some heat source is found that can accommodate the X-ray spectral measurements successfully, it must be one of the major (unseen!) energy flows in the Universe! Whilst the luminosity of a cooling flow may be only 10 per cent of the total cluster $\mathrm{X}$-ray luminosity and the mass lost through cooling a negligible drain on the enormous outer atmosphere, the cooling luminosity is a major loss of energy from the cluster core. Whatever is eventually decided about cooling flows, they cannot be an insignificant process.

\section{3b Star Formation in Cooling Flows}

As already mentioned, the average mass of a star formed in a cooling flow must be considerably smaller than in our Galaxy. In particular, the fraction of the mass turned into massive $O B$ stars must be very small, since there is little ultraviolet light seen with the IUE (e.g. Fabian, Nulsen \& Arnaud 1984). (The shortest cooling times for the X-ray emitting gas $\left(\sim 3 \times 10^{7} \mathrm{yr}\right)$ are comparable to the lifetime of $B$ stars, so intermittency of the flow cannot be important here.) In understanding why there are these differences, it would be helpful to have a predictive theory of star formation for our Galaxy. As we do not, we must look for differences. Some are a) the lack of dust in gas that has cooled from $T>10^{7} \mathrm{~K}$ (Draine \& Salpeter 1979), which presumably means that there are no molecular clouds such as give birth to massive stars in our Galaxy; b) the thermal pressure of the gas is $100-1000$ times higher than in our interstellar medium and that affects the Jeans mass; c) differential motions, cloud masses, angular momentum and magnetic field strengths may be different.

The general statement about the necessity for low mass stars applies to the bulk of the cooled gas. In the centre, there are often seen optical emission line blobs or filaments, which may be atypical of most of the flow. These blobs may give rise to higher mass stars. There is some excess blue light observed at the centres of many cooling flows and it does correlate in strength with the mass deposition rate (Johnstone, Fabian \& Nulsen 1987). Spectral fits of the blue light together with upper limits from IUE spectra show that the upper mass limit for stars must be around $1.5-2 \mathrm{M}_{\odot}$ there. Some F and early $\mathrm{G}$ stars are seen. Of course, the best place to look for the bulk of the cooled gas is at large radii where the underlying stellar light of the galaxy is least, so the contrast is highest.

\section{3c The Behaviour of Gas Blobs}

The distributed manner of the mass deposition shows that the cooling flow is inhomogeneous. This means that it contains blobs of gas that are denser than the surrounding gas. How these blobs behave is ill-understood. Malagoli et al. (1987) and Balbus (1988) have shown that cooling flows are not expected to be thermally unstable and so cannot generate sizable blobs from initially infinitesimal perturbations. A region that is slightly overdense 
with respect to its surroundings will fall ahead of the flow under gravity and join a region of similar properties to itself.

Nulsen (1986) has pointed out that the gravitationally-induced motions of a blob relative to its surroundings will cause it to break up and so increase its area-to-mass ratio and have a lower terminal velocity. Magnetic fields can then help to 'pin' a blob to the flow so that it can become unstable.

Another likely property of a flow is turbulence, or at least chaotic motions (Loewenstein \& Fabian 1988; Pringle 1988). This can stir the gas around and reduce the tendency of dense blobs to flow inward. The motion of cluster galaxies, of subcluster infall and of the flow itself can all promote chaotic motions. Turbulence of the hot gas can also explain the large velocity spread seen in the optical line-emitting filaments and blobs common at the centres of flows. It can also help to heat the cold blobs. Future high-resolution $\mathrm{X}$-ray spectroscopy can test this idea.

\section{Optical Evidence for Cooling Flows}

The presence of optical emission-line filaments and blobs in many cooling flows (note that some, e.g. A2029, do not have any detectable emission) can be used as a diagnostic of the conditions in those flows. We can also use them to identify candidate cooling flows where $\mathrm{X}$-ray observations are not available or at higher redshifts if there is some resemblance to nearby flows.

One property that can be obtained from the optical spectra is the gas pressure, in particular from the [SII] lines. In the Perseus cluster, these lines change their ratio indicating high density and thus high pressure within $5 \mathrm{kpc}$ of the nucleus (Johnstone \& Fabian 1988). Since the pressure has risen above the $\mathrm{X}$-ray inferred pressure (from the $\mathrm{X}$-ray surface brightness) at $20 \mathrm{kpc}$, the mean gas temperature must be down to the virial temperature of the central galaxy, NGC1275, of about $10^{7} \mathrm{~K}$. This is further confirmation that the gas has cooled there below the outer temperature of $\sim 8 \times 10^{7} \mathrm{~K}$. Since the gas pressure is so high there, the magnetic pressure cannot be more than about twice the gas pressure (and is probably less than that).

The velocity spread of the optical lines does also indicate large chaotic motions in the hot gas, as discussed earlier. The origin of the optical line emission remains a problem. As already mentioned in $\S 3$, the cooling gas by itself cannot produce much detectable optical emission; some of the gas must be held at $\sim 10^{4} \mathrm{~K}$ in order that we do see the optical lines. This means that there is a distributed, weak heat source at the centres of many cooling flows (see.e.g. Johnstone \& Fabian 1988). The optical emission is typically less than $1 \%$ of $L_{\text {cool }}$ (and always less than $10 \%$ ) and so this heat source cannot significantly affect the cooling flow itself. It is likely that the turbulent energy of the cooling gas is the heat source.

The optical line emission of nearby flows shows some resemblances to the nebulosities surrounding many distant (3CR) radio galaxies and radio-loud quasars (Fabian et al.1986, Hintzen \& Romanishin 1986). One way to check whether they are indeed embedded in hot cooling gas is to measure the gas pressure. If the emission-line gas is very extended and has a pressure, $n T>10^{5} \mathrm{~cm}^{-3} \mathrm{~K}$, then it is likely to be confined, or it would rapidly disperse. The confining hot gas is then part of a cooling flow unless its temperature exceeds $5 \times 10^{7} \mathrm{~K}$. The arguments against either unconfined or gravitationally confined gas are discussed in detail in Fabian et al.(1987).

The pressure of gas around quasars cannot easily be measured from the [SII] lines, which are faint and only change where the pressure is very high close to the centre. We have pursued a photoionization method which assumes that the ionization state of, say, oxygen is due to the competition between photionization by UV radiation from the quasar 


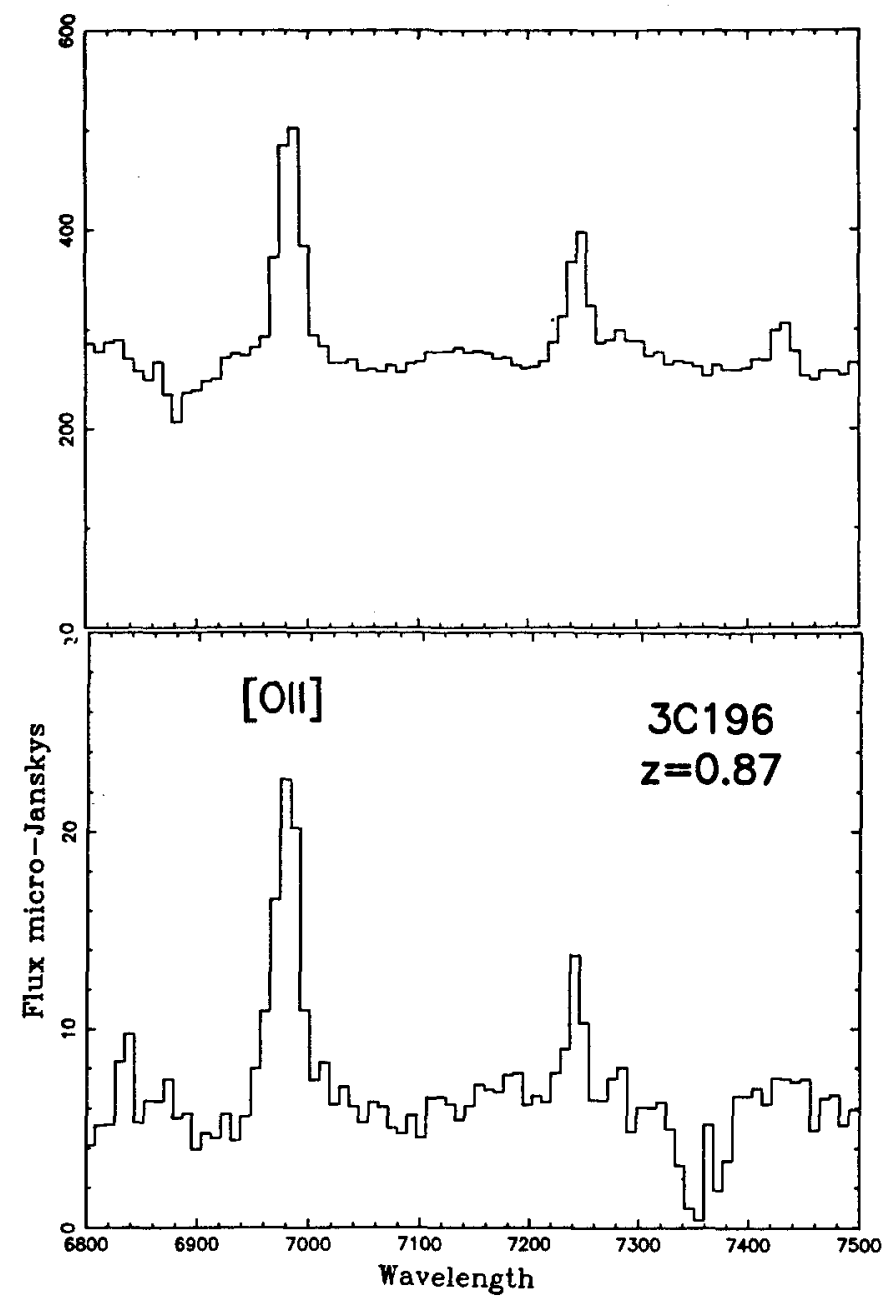

Figure 5. Optical spectra of the radio-loud quasar 3C196 on-nucleus (top panel) and 3 arcsec off-nucleus (bottom panel) showing the increased equivalent width of the [OII] line off-nucleus. Comparison of the [OIII] and [OII] emission shows that the surroundings of $3 \mathrm{C} 196$ are at high gas pressure, consistent with $\mathrm{a} \sim 100 \mathrm{M}_{\odot} \mathrm{yr}^{-1}$ cooling flow (Crawford 1988).

nucleus and recombination. The higher the gas pressure and so density, the more rapid will be the recombination and so the lower will be the ionization state. Basically, if we use the forbidden oxygen lines to measure the ionization state of the gas,

$$
\frac{[\mathrm{OIII}]}{[\mathrm{OII}]} \propto f\left(\frac{L_{\text {ion }}}{n R^{2}}\right),
$$

where the function $f$ is obtained by computation (using G. Ferland's code), $L_{\text {ion }}$ is the 
ionizing luminosity of the quasar nucleus, obtained from an interpolation between the observed IUE and X-ray emission, $R$ is the radial distance of the nebulosity where the intensity ratio of [OIII] to [OII] line is measured and $n$ is the gas density. The gas pressure is then $\sim 10^{4} n \mathrm{~cm}^{-3} \mathrm{~K}$. We have found that $3 \mathrm{C} 48$ at redshift $z=0.37$ and several other radioloud quasars out to $z=0.87$ have pressures indicating surrounding cooling flows with $\dot{M} \sim 100 \mathrm{M}_{\odot} \mathrm{yr}^{-1}$. There is other evidence that radio-loud quasars lie in clusters and groups from galaxy counts around the quasars (Yee \& Green 1984).

This work suggests that cooling flows can have been more common in the past and possibly more massive, if the high line luminosities of the distant 3CR galaxies (e.g. Spinrad \& Djorgovski 1984) may be scaled up from NGC1275. The cooling flows surrounded the dominant galaxies in groups and poor clusters, which have since merged with other groups to form the present day clusters (Fabian 1988). Some of the evolution of radio-loud quasars and of radio sources may be due to the evolution of their surrounding cooling flows.

\section{What Next?}

There is much left to do. On the theoretical side there are many unsolved problems on the behaviour of blobs and on how the flow became, or becomes, inhomogeneous. What are the length scales involved? How does the star formation take place? How is conduction suppressed? On the observational side we clearly need to find the cooled gas in some form, hopefully as stars. This can be achieved with sensitive searches for an $r^{-1}$ surface brightness profile at near IR wavelengths.

Of greatest need are more X-ray images and more X-ray spectra. ROSAT, BBXRT, ASTRO-D and AXAF will supply them. Spatially-resolved, high-resolution spectroscopy is the ultimate goal. Then we can tell whether cooling flows really do have something to do with galaxy formation in general.

\section{Acknowledgements}

I thank Roderick Johnstone for help and the Royal Society for supporting my work.

\section{References}

Arnaud, K.A., 1988. In Cooling Flows in Clusters and Galaxies, ed. A.C.Fabian, Reidel, 31.

Balbus, S., 1988. Astrophys. J., 328, 395.

Bertschinger, E. \& Meiksin, A., 1986. Astrophys.J, 306, L1.

Bregman, J.D. \& David L.P., 1988. Astrophys. J., 326, 639.

Canizares, C.R., Clark, G.W., Markert, T.H., Berg, C., Smedira, M., Bardas, D., Schnopper,

H. \& Kalata, K., 1979, Astrophys. J., 234, L33.

Canizares, C.R., 1981. In $X$-ray Astronomy with the Einstein Satellite ed. R. Giacconi, Reidel, 215.

Canizares, C.R., Clark, G.W., Jernigan, J,G. \& Markert, T.H., 1982. Astrophys. J., 262, L33.

Canizares, C.R., Stewart, G.C. \& Fabian A.C., 1983. Astrophys. J., 272, 449.

Canizares, C.R., Markert, T.H. \& Donahue, M.E., 1988. In Cooling Flows in Clusters and Galaxies, ed. A.C.Fabian, Reidel, 63. 
Crawford, C.S., 1988. PhD Thesis, University of Cambridge.

Crawford, C.S., Arnaud, K.A., Fabian, A.C. \& Johnstone, R.M., 1989. Mon. Not. R. astr. Soc., 236, 277.

Fabian, A.C. et al., 1984. Astrophys. J., 189, L59.

Fabian, A.C., Hu, E.M., Cowie, L.L \& Grindlay, J.,1981. Astrophys. J., 248, 47.

Fabian, A.C., Nulsen, P.E.J. \& Canizares, C.R., 1982. Mon. Not. R. astr. Soc., 201, 933.

Fabian, A.C., Nulsen, P.E.J. \& Canizares, C.R., 1984. Nature, 311, 733.

Fabian, A.C., Nulsen, P.E.J. \& Arnaud, K.A., 1984. Mon. Not. R. astr. Soc., 208, 179.

Fabian, A.C., Arnaud, K.A., Nulsen, P.E.J. \& Mushotzky, R.F., 1986. Astrophys. J., 305, 9.

Fabian, A.C., Arnaud, K.A. \& Thomas, P.A., In Dark Matter in the Universe, eds. J. Kormendy \& G.R. Knapp, Reidel, 201.

Fabian, A.C., Crawford, C.S., Johnstone, R.M. \& Thomas, P.A., 1987. Mon. Not. R. astr. Soc., $228,963$.

Fabian, A.C., 1988. In Cooling Flows in Clusters and Galaxies, ed. A.C.Fabian, Reidel, 315.

Fabian, A.C., 1988. In Hot Thin Plasmas in Astrophysics, ed. R. Pallavicini, Reidel, 293.

Gorenstein, P., Fabricant, D., Topka, K., Tucker, W. \& Harnden, F.R., 1977. Astrophys. $J ., 216$, L95.

Helmken, H., Delvaille, J.P., Epstein, A., Geller, M.J., Schnopper, H.W. \& Jernigan, J.G., 1978. Astrophys. J., 221, L43.

Hintzen, P. \& Romanishin, W., 1986. Astrophys. J., 311, L11.

Henry, J.P \& Henriksen, M.J., 1986. Astrophys. J., 301, 689.

Johnstone, R.M., Fabian, A.C. \& Nulsen, P.E.J., 1987. Mon. Not. R. astr. Soc., 224, 75.

Johnstone, R.M. \& Fabian, A.C., 1987. Mon. Not. R. astr. Soc., 233, 581.

Jones, C. \& Forman, W., 1984. Astrophys. J., 276, 38.

Lea, S.M., Mushotzky, R.F. \& Holt, S.S., 1982. Astrophys. J., 262, 24.

Loewenstein, M. \& Fabian, A.C., 1988. Preprint.

Malagoli, A., Rosner, R. \& Bodo, G., 1987. Astrophys. J., 319, 632.

Miller, L., 1986. Mon. Not. R. astr. Soc., $220,713$.

Mitchell, R.J., Charles, P.A., Culhane, J.L., Davison, P.J.N. \& Fabian, A.C., 1975. Astrophys. J., 200, L5.

Mould, J.R., Oke, J.B. \& Nemec, J.M., 1987. Astr. J., 92, 53.

Mushotzky, R.F., Holt, S.S, Smith, B.W., Boldt, E.A. \& Serlemitsos, P.J., 1981. Astrophys. $J ., 244$, L47.

Mushotzky, R.F. \& Szymkowiak, A.E. , 1987b. In Cooling Flows in Clusters and Galaxies, ed. A.C.Fabian, Reidel, 47.

Nulsen, P.E.J., 1986. Mon. Not. R. astr. Soc., $221,377$.

Nulsen, P.E.J., 1988.In Cooling Flows in Clusters and Galaxies, ed. A.C.Fabian, Reidel, 378.

Pringle, J.E., 1988. Preprint.

Sarazin, C.L. \& O'Connell, R.W., 1983. Astrophys. J., 258, 552.

Schwartz, D.A., Schwarz, J. \& Tucker, W.H., 1980. Astrophys. J., 238, L59.

Silk, J., Djorgovski, G., Wyse, R.F.G. \& Bruzual, G.A., 1986. Astrophys. J., 307, 415.

Singh, K.P., Westergaard, N.J. \& Schnopper, H.W., 1986. Astrophys. J., 308, L51.

Spinrad, H. \& Djorgovski, G., 1984. Astrophys. J., 280, L9.

Stewart, G.C., Canizares, C.R., Fabian, A.C. \& Nulsen, P.E.J., 1984a. Astrophys. J., 278, 536.

Stewart, G.C., Fabian, A.C., Jones, C. \& Forman, W., 1984b. Astrophys. J., $285,1$.

Thomas, P.A., Fabian, A.C. \& Nulsen, P.E.J., 1987. Mon. Not. R. astr. Soc., 228, 973.

Tucker, W.H. \& Rosner, R., 1982. Astrophys. J., 267, 547. 
White, R.E. \& Sarazin, C.L., 1987. Astrophys. J., 318, 612, 621, 629.

Yee, H.K.C. \& Green, R.F., 1984. Astrophys. J., $280,79$.

\section{DISCUSSION.}

A. Hamilton. How important is a finite metallicity to a cooling flow? Is a zero metallicity flow modified in any interesting way?

A.C. Fabian. Not particularly, most of the cooling is by bremsstrahlung above $10^{7} \mathrm{~K}$.

S.A. Balbus. You mentioned that some $50 \%$ of clusters show cooling flows, according to Arnaud's sample. What was the selction criterion used for picking the sample members?

A.C. Fabian. They are the better observed clusters with more than a few hundred counts. They tend to be round and peaked. Christine Jones has studied a wider sample and suggests a value nearer $30 \%$.

G.S. Bisnovatyi-Kogan. How can the enrichment of intergalactic gas in the clusters occur in the presence of cooling flows? Is it necessary to have the flow switched-off by a very luminous object, observed at higher redshift?

A.C. Fabian. Much of the enrichment in nearby clusters presumably occurred long ago and from the cluster galaxies, not just the central one. For galaxy formation, the cooling flow may well be a mess, although enrichment may still occur first (see Fabian et al., Astrophys. J., 1986).

J.Grindlay. For $3 \mathrm{C} 196$, at $\mathrm{z}=0.87$, if the inferred cooling flow was $\dot{M} \sim 100 \mathrm{M}_{\odot} \mathrm{yr}^{-1}$, the responsible galaxy cluster (supplying the flow) must be large enough to be detected in deep optical images. Is there evidence for this?

A.C. Fabian. I'm not aware that anyone has looked. The cluster may be poor (cf. MKW 3s). Yee \& Green $(1984,87)$ find that radio-loud quasars are in groups and clusters at $z<0.6$, but that they are difficult to detect at higher $z$.

F. Winkler. How severely should observations with the present IR arrays be able to constrain the Mass Function for the posited stars resulting from cooling flows?

A.C. Fabian. Interesting results are possible since the stars from the cooling flow have $\rho_{*} \propto r^{-2}$, whereas the visible galaxy halo drops off more steeply. We hope to take suitable IR images early next year at UKIRT.

H. Tananbaum. You stated that cooling flows may be able to account for the dark matter in clusters (or cluster cores) in a baryonic form (i.e. low-mass stars). At present rates the cooling flows would only be able to account for a small fraction of the 'observed' (or measured) dark matter. Do you think that cooling flow rates were higher in the past, or that 2 forms/types of dark matter exist, or both?

A.C. Fabian. The simplest implication from the present formation of some dark matter by cooling flows is that cooling flows were stronger in the past. I'm not yet sure that non-baryonic matter is absolutely required. 